\title{
2019 List of Reviewers
}

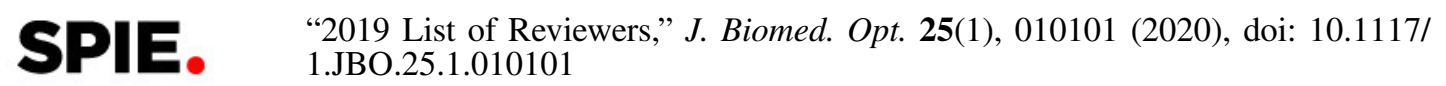


The Journal of Biomedical Optics sincerely thanks the following individuals who served as reviewers in 2019. The success of our publication hinges on the voluntary contributions of time and energy put forth by these professionals.

Atefeh Abdolmanafi Ibrahim Abdulhalim Walter Akers

Prabhu Britto Albert Sergey Alexandrov Robert Amelard Amirhessam Aminfar Joseph Angelo, Jr Md Ansari

Matthew Applegate

Vanderlei Bagnato

Wesley Baker

Oluwaseyi Balogun

Mihaela Balu

Timothy Baran

Utku Baran

Jennifer Barton

Alexey Bashkatov

Adam Bauer

Peter Baumhoff

Julien Bec

Muyinatu Bell

Paul Bernstein

Lina Bezdetnaya Bolotine

Olga Bibikova

Kostadinka Bizheva

Richard Blackmon

Ekaterina Borisova

Richard Bouchard

Nada Boustany

Audrey Bowden

Alan Boyde

Adrian Bradu

John Briers

Ralf Brinkmann

Peter Burgholzer

David Busch

Tyler Bywaters Rice

Delia Cabrera DeBuc

Fuhong Cai

Paul Campagnola

Kirby Campbell

Murat Canpolat

$\mathrm{Xu}$ Cao

Juliana C. Carmello

Hongwei Chen

Jyh-Cheng Chen

Mingzhou Chen

Nanguang Chen

Sungliang Chen

Ye Chen

Yu Chen

Shau Poh Chong

Ken Chu

Zhongdi Chu

Joseph Chue-Sang

\section{Sohyun Chung \\ Riccardo Cicchi \\ Olga Conde \\ Lorenzo Cortese \\ Andrea Curatolo}

Anna Cysewska-Sobusiak

Anabela Da Silva

Michael Daly

Arash Darafsheh

Hadis Dashtestani

Rupsa Datta

Scott C. Davis

Richard Day

Catharina de Lange Davies

Ana de Paula

Frederico de Sousa

Hamid Dehghani

Michele Diana

Jouke Dijkstra

Yichen Ding

Chen-Yuan Dong

Alexander Doronin

Dane Drutis

Nicholas Durr

Daniel Elson

Stanislav Emelianov

Mohsen Erfanzadeh

Thomas Ertl

Rinat Esenaliev

Karen Esmonde-White

Conor Evans

Leyuan Fang

Qianqian Fang

Qiyin Fang

Felix Fanjul-Velez

Hamid Farrokhi

Baowei Fei

Shangyuan Feng

Ting Feng

Pietro Ferraro

Reto Fiolka

Jonathan Fisher

Blaise Frederick

Ingemar Fredriksson

Paul French

Daniel Fried

Nathaniel Fried

Ling Fu

Ekaterina Galanzha

Xiaosong Gan

Grigory Gelikonov

Elina Genina

Irene Georgakoudi

Arkadiusz Gertych

Eugen Gheorghiu

Michael Giacomelli
Emily Gibson

Natalia Gladkova

Adam Glaser

Craig Goergen

Borivoj Golijanin

James Grant-Jacob

Bruno Grassi

Kate Grieve

Dirk Grosenick

Meisha Gul

Hanwen Guo

Abhijit Gurjarpadhye

Steffen Hackbarth

Nathan Hagen

Michael Hamblin

Songfeng Han

Diane Harper

Valentina Hartwig

Nathaniel Haven

Carole Hayakawa

Joseph Hayward

Wido Heeman

Christine Hendon

Matt Hepburn

Taylor Hinsdale

Kenneth Hoffmann

Martin Hofmann

Vanessa Holanda

Christopher Hoy

Chenfei Hu

Song $\mathrm{Hu}$

Huangchiao Huang

Xiangrun Huang

Yong Huang

Anne Humeau-Heurtier

Jeeseong Hwang

Nicusor Iftimia

Justus Ilgner

Michael Insana

M. Shahidul Islam

Steven Jacques

Michael Jaeger

Mengyu Jia

Jingying Jiang

Shudong Jiang

Janggun Jo

Jesse V. Jokerst

Takashi Kakue

Vyacheslav Kalchenko

Mikhail Kandel

Dimitris Kapsokalyvas

Sebastian Karpf

Kavon Karrobi

Gordon Kennedy

Siddharth Khare

Nikolai Khlebtsov 


\begin{tabular}{|c|c|c|}
\hline Alexander Khmaladze & Barry Masters & Samuel Powell \\
\hline Aaron Kho & Lev Matveev & Scott Prahl \\
\hline Alwin Kienle & David McGloin & Manojit Pramanik \\
\hline Chulhong Kim & Trevor McKee & $\mathrm{KPu}$ \\
\hline Ki Hean Kim & Mike McShane & Le Qiu \\
\hline Myung Kim & Andrei Medvedev & Kyle Quinn \\
\hline Karsten Koenig & Mohammad & Narasimhan Rajaram \\
\hline Kivanc Kose & Mehrmohammadi & Gabriel Ramirez \\
\hline Sri-Rajasekhar Kothapalli & Jerome Mertz & Julio Ramirez-San Juan \\
\hline Stefan Kroll & Rickson Mesquita & Janaka Ranasinghesagara \\
\hline Yoshiaki Kubota & Kirk Michaelian & Fulvio Ratto \\
\hline Manoj Kumar & Daniel Milej & Aniruddha Ray \\
\hline Cristina Kurachi & Takeo Minamikawa & Karen Reiser \\
\hline David Kwartowitz & Andreia Moco & Liqiang Ren \\
\hline Sun II Kwon & Suman Mondal & Brendon Restall \\
\hline Sunkuk Kwon & Claudia Mota & Rebecca Richards-Kortum \\
\hline Lily Lai & Judith Mourant & Jose Rico-Jimenez \\
\hline Horacio Lamela Rivera & Timothy Muldoon & Jorge Ripoll \\
\hline Guy Lamouche & Oleg Nadyarnykh & Charles Riva \\
\hline Lu Lan & Florent Nageotte & Yair Rivenson \\
\hline Joseph Landry & Eman Namati & William Roach \\
\hline Pierre Lane & Sreyankar Nandy & Michael Roberts \\
\hline Birgit Lange & Kamran Nasiriavanaki & Francisco Robles \\
\hline Kirill Larin & Andrew Nattestad & Darren Roblyer \\
\hline Ethan LaRochelle & John Quan Nguyen & Jeremy Rogers \\
\hline Jan Laufer & Tan Nguyen & Peter Rolfe \\
\hline Du Le & Addison Niemeyer & Marek Romanowski \\
\hline Martin Leahy & Bo Ning & Joseph Rosen \\
\hline Hsiang-Chieh Lee & Santi Nonell & Francesca Rossi Paoletti \\
\hline Robert Lee & Thomas O'Sullivan & Christopher Rowlands \\
\hline Seung Yup Lee & Yusuke Ogura & Marcello Rubessa \\
\hline Terence Leung & Masato Ohmi & Alberto Ruiz \\
\hline Buhong Li & Jeffrey Oliver & Rolf Saager \\
\hline Changhui Li & Elizabeth Olson & Leonardo Sacconi \\
\hline Jiao Li & Yihong Ong & Negar Sadeghipour \\
\hline Lei Li & Felipe Orihuela-Espina & Andrey Sagaidachyi \\
\hline Li Li & Daniel Orringer & Sudipta Saha \\
\hline Qingli Li & Cynthia Pagba & Sava Sakadzic \\
\hline Rongguang Liang & Akilan Palanisami & Inga Saknite \\
\hline Antonia Lichtenegger & Nikhil Paliwal & Felix Scholkmann \\
\hline Adam Liebert & Guenther Paltauf & Karl Schulmeister \\
\hline Lothar Lilge & Vimal Prabhu Pandiyan & Hinnerk Schulz-Hildebrandt \\
\hline Norman Lippok & Asael Papour & Dietrich Schweitzer \\
\hline Liu Liu & Seonyeong Park & Oxana Semyachkina- \\
\hline Rui Liu & YongKeun Park & Glushkovskaya \\
\hline Yang Lou & Manish Patankar & Natan Shaked \\
\hline Heard Lowry & Ronak Patel & Vladislav Shcheslavskiy \\
\hline Guolan Lu & Ivan Pelivanov & Jennifer Shell \\
\hline Rongwen Lu & Zhaoqiang Peng & Yihui Shen \\
\hline Alfredo Lucas & Rozhin Penjweini & Lingyan Shi \\
\hline Andrei Lugovtsov & Daqing Piao & Marina Shirmanova \\
\hline Jianwen Luo & Fabien Picot & Xiao Shu \\
\hline Cheng Ma & Mark Pierce & Mira Sibai \\
\hline Steen Madsen & Roberto Pilot & Ludovico Silvestri \\
\hline Sudipta Maiti & Michael Pircher & Kanwarpal Singh \\
\hline Boris Majaron & Andrew Plumb & Emil Sobol \\
\hline Dolonchampa Maji & Brian Pogue & Sergei Sokolovski \\
\hline Srivalleesha Mallidi & Daria Pominova & Cheol Song \\
\hline Benjamin Maloney & Alexey Popov & Janis Spigulis \\
\hline Amir Manbachi & Dmitry Postnov & Antonello Spinelli \\
\hline Dominik Marti & Eric Potma & Bryan Q. Spring \\
\hline
\end{tabular}


2019 List of Reviewers

Vivek Srinivasan

Ronald Sroka

Samuel Streeter

lain Styles

Klaus Suhling

Chiawei Sun

Yuansheng Sun

Ulas Sunar

Nima Tabatabaei

Yidong Tan
Jianbo Tang

Yubo Tang

Chao Tao

Tanja Tarvainen 